\title{
Image Registration Using Threefold Orthogonal Wavelet
}

\author{
Smadar Gefen ${ }^{* a}$, Oleh Tretiak ${ }^{\mathrm{b}}$, and Jonathan Nissanov ${ }^{\mathrm{a}}$ \\ ${ }^{a}$ Computer Vision Laboratory for Vertebrate Brain Mapping, Department of Neurobiology and \\ Anatomy, Drexel University College of Medicine, 2900 Queen Lane \\ Philadelphia, PA 19129 \\ ${ }^{\mathrm{b}}$ Electrical and Computer Engineering Department, Drexel University, 3141 Chestnut St. \\ Philadelphia, PA 19104
}

\begin{abstract}
An algorithm for multidimensional nonlinear registration is proposed. The deformation field between two elastic bodies is represented by a multi-resolution separable wavelet. Using a progressive approach that reduces algorithm complexity the registration parameters are recovered in a coarse to fine order. A custom wavelet that approximates threefold orthogonality is developed. The performance of the algorithm is evaluated by the alignment of sections from mouse brains. The wavelet registration algorithm demonstrated on average fourfold improvement in section alignment over linear alignment.
\end{abstract}

Keywords: Elastic deformation, nonlinear registration, threefold orthogonal wavelet, segmentation, mouse brain, spatial normalization.

\section{INTRODUCTION}

Registration of images is desirable whenever a comparison between two complementary dataset is required. Medical images alignment is an especially challenging problem due to nonlinear spatial intersubject variability and due to the lack of point-to-point correspondence. The improved resolution of imaging systems and the resulting large data volumes further complicate the registration problem. In this study, a progressive approach toward image registration is proposed. It is a wavelet-based, surface-guided, scalable algorithm for multidimensional image registration. The method is evaluated using histologial murine brain sections.

The model used in this study regards the tissue to be aligned as if it was an elastic medium that is subject to the static Navier Partial Differential Equation (PDE) from continuum mechanics. This image registration model was pioneered by Bajcsy and by Broit [1,2]. A separable wavelet was used to represent the deformation field which was modeled as a combination of components including global deformation - represented by the lowest resolution level of the wavelet decomposition - and local deformation details - represented by the higher resolution levels of the wavelet decomposition. The wavelet coefficients that correspond to each different signal component were estimated separately and progressively, starting from global deformation down to local deformation. This progressive approach for recovering the deformation signal is enabled by employing a threefold orthogonal wavelet. The registration parameters were estimated by minimizing the elastic energy (which is equivalent to solving for the static homogenous Navier PDE) as well as by minimizing the sum of squared distances between corresponding surfaces (which is equivalent to imposing boundary conditions).

Wavelets have been previously employed in image registration, either in order to represent the non-linear deformation between two images [3-5] or to represent the voxel intensity [6]. For example, Amit [3] used a wavelet basis as well as a Fourier basis to represent the deformation. He used a gradient descent optimization method to minimize the mean of squared distances between intensities of overlapping voxels. Similarly, Downie [4] used a simulated annealing optimization method to find the wavelet coefficients that minimize the sum of squared distances between intensities of corresponding voxels. Both are image-based and as such are most suited for applications dealing with intra-modality datasets. In contrast, the registration algorithm developed in this study is based on geometric features: alignment is guided by samples from the objects' surfaces and therefore can be used for applications dealing with inter- as well as intra- modality scans.

\footnotetext{
*sgefen@drexel.edu; phone 1215 991-8455; fax 1 215-843-9367; www.drexel.edu
} 
The proposed surface-guided method compensates for nonlinear deformation that is represented by a wavelet multiresolution decomposition. The similarity metric used is based on the sum of squared distances where a distance is the interval between a point on the test surface and the closest point to it on the reference surface. Alignment is achieved employing a progressive version of the Marquardt-Levenberg (M-L) optimization algorithm that minimizes a functional. This functional is the sum of two terms, namely the sum of squared distances and the elastic energy. While the alignment algorithm formulated here is a two-dimensional one (alignment that is guided by the structures' contours instead of by the structures' surfaces), its generalization to a three-dimension one is straightforward. The performance of the algorithm was demonstrated for the 2D case of mouse brain mapping focusing on the olfactory bulbs, a brain area where sensory information from the nose is processed. In Section 2, the proposed registration algorithm is presented. Section 3 describes the development of the threefold orthogonal wavelet that was used in this study. Section 4 demonstrates the algorithm as applied to the registration of sections from the murine olfactory bulbs. Section 5 concludes with a discussion.

\section{ALGORITHM DESCRIPTION}

Nonlinear registration is the operation of recovering the deformation field, $\mathbf{u}(\mathbf{x}, \mathbf{c})$, that maps a test object to a reference object as follows: $x^{\prime}=x+u^{1}(x, y ; \mathbf{c})$ and $y^{\prime}=y+u^{2}(x, y ; \mathbf{c})$, where $(x, y)$ are coordinates in the test object space and $\left(x^{\prime}, y^{\prime}\right)$ are the corresponding coordinates in the reference object space. With the developed algorithm the deformation field is recovered by an optimization procedure that searches for the registration parameter vector, $\mathbf{c}$, that minimize: (1) the sum of squared Euclidian distances between corresponding points on the test object's contours and points on the corresponding reference object's contours and (2) the elastic energy of the deformation. Thus, a functional $E(\mathbf{c})=e(\mathbf{c})+\omega \cdot L(\mathbf{c})$ is minimized, where $e(\mathbf{c})$ is the sum of squared Euclidian distances between corresponding points, $L(\mathbf{c})$ is the elastic energy, and the parameter $\omega$ is a weight constant that is empirically determined. We next detail the proposed wavelet-based registration algorithm and the progressive Marquardt-Levenberg-based optimization procedure employed.

The two-dimensional separable wavelet decomposition of a deformation field, $\mathbf{u}(\mathbf{x})$, everywhere within a rectangular support $\mathbf{N}=\left(N_{x}, N_{y}\right)$ is given by:

$$
u(\mathbf{x})^{i}=\sum_{\mathbf{k}=0}^{\mathbf{N}_{J}-1} 2^{-J} c_{J \mathbf{k}}^{i 1} \Phi^{1}\left(2^{-J} \mathbf{x}-\mathbf{k}\right)+\sum_{j=R}^{J} 2^{-j} \sum_{s=2}^{4} \sum_{\mathbf{k}=0}^{\mathbf{N}_{j}-1} c_{j \mathbf{k}}^{i s} \Phi^{s}\left(2^{-j} \mathbf{x}-\mathbf{k}\right)
$$

where $i$ is an index denoting the two directions, $x$ and $y$, of the vector function $\mathbf{u}(\mathbf{x}), s$ is an index denoting the subband, $j$ is an index denoting the resolution levels, and $\mathbf{k}=\left(k_{x}, k_{y}\right)$ is the translational index within the $\mathbf{N}_{j} \equiv 2^{-j} \mathbf{N}=2^{-j}\left(N_{x}, N_{y}\right)$ support. Hence, each wavelet coefficient $c_{j \mathbf{k}}^{i s}$ is indexed by the direction $(i)$, the orientation $(s)$, the resolution $(j)$, and the spatial location ( $\mathbf{k})$ it stands for. The basis functions, $\Phi_{j \mathbf{k}}^{s} \equiv \Phi^{s}\left(2^{-j} \mathbf{x}-\mathbf{k}\right)$, are a tensor product of the one-dimensional scaling and wavelet functions as follows:

$$
\begin{aligned}
& \Phi^{1}=\phi\left(2^{-j} x-k_{x}\right) \phi\left(2^{-j} y-k_{y}\right) \\
& \Phi^{2}=\phi\left(2^{-j} x-k_{x}\right) \psi\left(2^{-j} y-k_{y}\right) \\
& \Phi^{3}=\psi\left(2^{-j} x-k_{x}\right) \phi\left(2^{-j} y-k_{y}\right) \\
& \Phi^{4}=\psi\left(2^{-j} x-k_{x}\right) \psi\left(2^{-j} y-k_{y}\right)
\end{aligned}
$$

Note that since we assume only that $\mathbf{u}(\mathbf{x})$ is a smooth deformation - the elastic deformation is in $L^{2}-$ the representation in (1) is merely an approximation. The accuracy of this approximation is controlled by our choice of the index $R$ that ranges here between 1 and $J$, where $2^{J+1}=\min \left(N_{x}, N_{y}\right)$. Consequently, for example, in the case where 
$N_{x}=N_{y}$ the number of parameters, $N_{c}$, required to define a deformation in one direction is $N_{c} \equiv 4 \cdot 2^{2(J-R+1)}$. As mentioned above, these parameters were estimated so that a functional $E(\mathbf{c})$ is minimized.

The first term of the functional, $E(\mathbf{c})$, that is being minimized is the sum of squared contour distances $e(\mathbf{c})$ :

$$
e(\mathbf{c})=\sum_{m=1}^{M} d_{m}^{2} \cong \sum_{m=1}^{M} d\left(\mathbf{a}\left(\mathbf{x}_{m}, \mathbf{c}\right), \mathbf{x}_{m}^{\prime}\right)^{2}
$$

where the contour distance, $d_{m}$, is the Euclidean distance between an already mapped point from the test contour, $\mathbf{a}\left(\mathbf{x}_{m} \in \mathrm{S}_{\text {test }}, \mathbf{c}\right)$ and the closest point to it on the reference contour, $\mathbf{x}_{m}^{\prime} \in S_{r e f}$. Similarly, a vector distance is defined as $\mathbf{v}_{m} \equiv \mathbf{a}-\mathbf{x}_{m}^{\prime}$. Using a Taylor expansion, it was shown in [7] that:

$$
e(\mathbf{c}) \approx e\left(\mathbf{c}_{l}\right)+\Delta^{T} \mathbf{b}+\Delta^{T} \mathbf{A} \Delta
$$

where $\mathbf{b}_{p} \equiv 2 \sum_{m=1}^{M} d_{m}\left(\mathbf{c}_{l}\right) \frac{\partial d_{m}\left(\mathbf{c}_{l}\right)}{\partial c_{p}}, A_{p q} \equiv \sum_{m=1}^{M} \frac{\partial d_{m}\left(\mathbf{c}_{l}\right)}{\partial c_{p}} \frac{\partial d_{m}\left(\mathbf{c}_{l}\right)}{\partial c_{q}}, \frac{\partial d_{m}\left(\mathbf{c}_{l}\right)}{\partial c_{j \mathbf{k}}^{i s}}=\frac{v_{m}^{i}}{d_{m}} \Phi^{s}\left(2^{-j} \mathbf{x}_{m}-\mathbf{k}\right)$, and $\Delta \equiv\left(\mathbf{c}-\mathbf{c}_{l}\right)$.

The second term, $L(\mathbf{c})$, that is being minimized is the following elastic energy:

$$
L(\mathbf{c})=\int\left[2\left(\frac{\partial u^{1}}{\partial x_{1}}\right)^{2}+\left(\frac{\partial u^{1}}{\partial x_{2}}\right)^{2}+\left(\frac{\partial u^{2}}{\partial x_{1}}\right)^{2}+2\left(\frac{\partial u^{2}}{\partial x_{2}}\right)^{2}+2 \frac{\partial u^{1}}{\partial x_{1}} \frac{\partial u^{2}}{\partial x_{2}}\right] d \mathbf{x}
$$

Substituting the signal as represented in (1) results in a linear combination of the integral:

$$
h_{p q}^{s t}(j, l, \mathbf{k}, \mathbf{n})=\int \frac{\partial \Phi^{s}\left(2^{-j} \mathbf{x}-\mathbf{k}\right)}{\partial x_{p}} \frac{\partial \Phi^{t}\left(2^{-l} \mathbf{x}-\mathbf{n}\right)}{\partial x_{q}} d \mathbf{x}
$$

where $p, q=1,2$. (An analytic evaluation of this integral is presented in [7].) In order to simplify the expression for the elastic energy we assume that the scaling and wavelet functions satisfy a principle we define and refer to here as threefold orthogonality. The threefold orthogonality property states that the scaling and wavelet functions are orthogonal to each other and orthogonal to each other's first and second derivatives:

$$
\int \phi^{\left(v_{2}\right)}(x) \cdot \psi^{\left(v_{2}\right)}(x-m) d x=0 \quad \text { or } \quad \int \phi^{(v)}(x) \cdot \psi(x-m) d x=0,
$$

where $m$ is an integer and $0 \leq v=v_{1}+v_{2} \leq 2$. The consequence of satisfying this property of threefold orthogonality is that the mixed terms of $h_{p q}^{s t}(j, l, \mathbf{k}, \mathbf{n})$ (terms with the basis functions from the different resolution levels $(j \neq l)$ and different orientations $(s \neq t))$ are canceled.

Let us continue and develop the expression in (5) for one component, $u^{i}(j, s)=\sum_{\mathbf{k}=0}^{\mathbf{N}_{j}-\mathbf{1}} c_{j \mathbf{k}}^{i s} \Phi^{s}\left(2^{-j} \mathbf{x}-\mathbf{k}\right)$, of the deformation field. In this case, (5) is a linear combination of the following terms:

$$
\int\left[\frac{\partial u^{i}}{\partial x_{p}} \frac{\partial u^{l}}{\partial x_{q}}\right] d \mathbf{x}=\sum_{\mathbf{n}} \sum_{\mathbf{k}} c_{j k}^{i s} c_{j n}^{l s} \int \frac{\partial \Phi^{s}\left(2^{-j} \mathbf{x}-\mathbf{k}\right)}{\partial x_{p}} \frac{\partial \Phi^{s}\left(2^{-j} \mathbf{x}-\mathbf{n}\right)}{\partial x_{q}} d \mathbf{x}=\sum_{\mathbf{n}} \sum_{\mathbf{k}} c_{j k}^{i s} c_{j n}^{l s} h_{p q}^{s s}(j, j, \mathbf{k}, \mathbf{n}) \equiv \mathbf{c}_{j}^{i s T} \mathbf{Q}_{j p q}^{s} \mathbf{c}_{j}^{l s} .
$$

Accordingly, the energy of the signal portion that corresponds to level $j$ and orientation $s$ is: 


$$
L_{j}^{s}\left(\mathbf{c}_{j}^{s}\right)=\left[\begin{array}{ll}
\mathbf{c}_{j}^{1 s T} & \mathbf{c}_{j}^{2 s T}
\end{array}\right] \cdot\left[\begin{array}{cc}
2 \mathbf{Q}_{j 11}^{s}+\mathbf{Q}_{j 22}^{s} & \mathbf{Q}_{j 12}^{s} \\
\mathbf{Q}_{j 12}^{s} & \mathbf{Q}_{j 11}^{s}+2 \mathbf{Q}_{j 22}^{s}
\end{array}\right] \cdot\left[\begin{array}{c}
\mathbf{c}_{j}^{1 s} \\
\mathbf{c}_{j}^{2 s}
\end{array}\right] \equiv \mathbf{c}_{j}^{s T} \mathbf{Q}_{j}^{s} \mathbf{c}_{j}^{s} .
$$

Hence the expression for the whole elastic energy is:

$$
L(\mathbf{c})=\mathbf{c}_{J}^{1 T} \mathbf{Q}_{J}^{1} \mathbf{c}_{J}^{1}+\sum_{j=R}^{J} \sum_{s=2}^{4} \mathbf{c}_{j}^{s T} \mathbf{Q}_{j}^{s} \mathbf{c}_{j}^{s} \equiv \mathbf{c}^{T} \mathbf{Q} \mathbf{c},
$$

where $\mathbf{Q}$ is a block diagonal matrix: $\mathbf{Q}=\operatorname{diag}\left(\mathbf{Q}_{J}^{1}, \mathbf{Q}_{J}^{2}, \mathbf{Q}_{J}^{3}, \mathbf{Q}_{J}^{4}, \mathbf{Q}_{J-1}^{2}, \mathbf{Q}_{J-1}^{3}, \mathbf{Q}_{J-1}^{4}, \ldots, \mathbf{Q}_{R}^{2}, \mathbf{Q}_{R}^{3}, \mathbf{Q}_{R}^{4}\right)$. Note though that the matrix $\mathbf{Q}$ depends only on the chosen support, $\mathbf{N}$, and the scaling and wavelet basis functions $\Phi_{j}^{s}$. Therefore, $\mathbf{Q}$ can be computed once offline and then used for the deformation estimation of any input object data. It can be shown that the matrix $\mathbf{Q}$ is linearly proportional to the Wavelet-Galerkin discretization matrix of the homogenous static Navier PDE [7]. This implies that minimizing the elastic energy is equivalent to solving the Navier PDE. Finally, the elastic energy in (10) is expressed in the vicinity of $\mathbf{c}_{l}$ using a Taylor series:

$$
L(\mathbf{c}) \cong \mathbf{c}_{l}^{T} \mathbf{Q} \mathbf{c}_{l}+\Delta^{T} 2 \mathbf{Q} \mathbf{c}_{l}+\Delta^{T} \mathbf{Q} \Delta
$$

and the expressions above for $e(\mathbf{c})$ and $L(\mathbf{c})$ are combined to result in the functional $E(\mathbf{c})$ :

$$
E(\mathbf{c})=e(\mathbf{c})+\omega \cdot L(\mathbf{c}) \approx e\left(\mathbf{c}_{l}\right)+\mathbf{c}_{l}^{T} \mathbf{Q} \mathbf{c}_{l}+\Delta^{T}\left(\mathbf{b}+2 \omega \mathbf{Q} \mathbf{c}_{l}\right)+\Delta^{T}(\mathbf{A}+\omega \mathbf{Q}) \Delta .
$$

Next, our objective is to iteratively search for $\Delta$, a step in the parameter space, progressing in a direction that minimizes $E(\mathbf{c})$. Following the M-L optimization method [8] this can be done by solving:

$$
\left(\mathbf{b}+2 \omega \mathbf{Q} \mathbf{c}_{l}\right)+[(\mathbf{A}+\omega \mathbf{Q})+\alpha \mathbf{I}] \Delta=0,
$$

where $\mathbf{I}$ is the identity matrix and $\alpha$ is a positive scalar. The smaller $\alpha$ is, the closer the M-L is to the Newton optimization method; the larger $\alpha$ is, the closer the M-L is to the gradient (steepest-descent) optimization method.

The optimization is done iteratively. At each iteration, first the vector $\mathbf{b}$ and the matrix $\mathbf{A}$ are computed based on the current spatial position of the test and the reference objects. Second, (13) is solved to yield a new update to the registration parameters. Lastly, the test object is mapped onto the reference object using updated registration parameters. Note that at each iteration, the contour distance $d_{m}$ needs to be computed for each point $\mathbf{x}_{m}$. This is done by searching through the set of points along the corresponding reference contour. This distance computation needs to be done at each iteration and for all the $\mathrm{M}$ test points. As a result, this distance computation is computationally expensive especially in the 3D case. Yet, using a distance map can reduce computation time significantly [9].

As mentioned before, our implementation of the optimization algorithm is a progressive one. We take advantage of the multi-resolution representation of the deformation and recover the wavelet parameters that define it in a coarse-tofine order. We first approximate the deformation with the wavelet decomposition, in (1), including only the lowest resolution level $(j=J)$ and the first orientation $(s=1)$. At this stage we find $\hat{\mathbf{c}}_{J}^{1}$ that minimizes $E\left(\mathbf{c}_{J}^{1}\right)$ following the M-L optimization method described above. Then we use $\hat{\mathbf{c}}_{J}^{1}$ to map the test image onto a new position that is now closer to the reference image. The residual deformation that still exists between the test and the reference images is then recovered using the rest of the wavelet decomposition components that represent the deformation "details" at its different resolutions and different orientations. Thus we proceed to find the parameters $\hat{\mathbf{c}}_{J}^{2}$ that minimizes $E\left(\mathbf{c}_{J}^{2}\right)$ and use now $\hat{\mathbf{c}}_{J}^{1}$ and $\hat{\mathbf{c}}_{J}^{2}$ to remap the test image toward the reference image. In the same manner we solve for the other two sub-bands, $s=2$ and $s=4$, at resolution level $j=J$. We continue down the wavelet pyramid to the next level of resolution $j=J-1$, solving for the different sub-bands at this level, $s=2,3,4$. Hence, the computation of the wavelet coefficients is done progressively until the estimation of all the coefficients is completed. 


\section{CUSTOM WAVELET DESIGN}

Threefold orthogonality of the wavelet bases simplifies significantly the definition of elastic energy - equation (10). Matrix $\mathbf{Q}$ becomes block diagonal so that a progressive solution of the problem is feasible. In this section we will develop a wavelet that approximates threefold orthogonality by using the lifting technique. The new, threefold orthogonal wavelet is found by obtaining the sequence $l(k)$ that satisfies the system $(20)$ in the least-squares sense.

In [10] Sweldens introduced the lifting method for the construction of wavelets with desired properties from any other biorthogonal wavelets. The idea behind the lifting scheme can be summarized as follows: Let $(\phi, \psi, \widetilde{\phi}, \widetilde{\psi})$ be a family of compactly supported biorthogonal scaling and wavelet functions associated with the filters $(\alpha, \beta, \widetilde{\alpha}, \widetilde{\beta})$, and let $l(k)$ be a finite sequence. A new family of biorthogonal scaling and wavelet functions $\left(\phi^{l}, \psi^{l}, \widetilde{\phi}, \widetilde{\psi}^{l}\right)$ is defined as:

$$
\begin{gathered}
\phi^{l}(t)=\sqrt{2} \sum_{k=-\infty}^{\infty} \alpha(k) \phi^{l}(2 t-k)+\sum_{k=-\infty}^{\infty} l(-k) \psi^{l}(t-k), \\
\psi^{l}(t)=\sqrt{2} \sum_{k=-\infty}^{\infty} \beta(k) \phi^{l}(2 t-k) \\
\widetilde{\psi}^{l}(t)=\widetilde{\psi}(t)-\sum_{k=-\infty}^{\infty} l(k) \tilde{\phi}(t-k) .
\end{gathered}
$$

Note that only $\widetilde{\phi}(t)$ does not change. Equation (16) is especially useful since it is the only one where the lifted function, $\widetilde{\psi}^{l}(t)$, depends only on the old functions, $\widetilde{\psi}(t)$ and $\widetilde{\phi}(t)$, as well as on the discrete series $l(k)$.

Let us take the $v$ derivative, $\frac{\partial^{v}}{\partial t^{v}}$, of (16):

$$
\widetilde{\psi}^{l(v)}(t)=\widetilde{\psi}^{(v)}(t)-\sum_{k=-\infty}^{\infty} l(k) \widetilde{\phi}^{(v)}(t-k)
$$

To ensure $v$-fold orthogonality (see (7) ) we require that

$$
\int \widetilde{\psi}^{l(v)}(t) \widetilde{\phi}(t-m) d t=0 .
$$

Substituting $\widetilde{\psi}^{l(v)}(t)$ from (17) in (18) leads to the equation:

$$
a_{21}^{v}(m)=\sum_{k=-\infty}^{\infty} l(k) a_{11}^{v}(m-k)
$$

where $a_{21}^{v}(m) \equiv \int \widetilde{\psi}^{(v)}(t) \widetilde{\phi}(t-m) d t$ and $a_{11}^{v}(m) \equiv \int \widetilde{\phi}^{(v)}(t) \widetilde{\phi}(t-m) d t$. Specializing this equation to our requirement, we see that to ensure threefold orthogonality we need a sequence $l(k)$ that satisfies 


$$
\begin{aligned}
& a_{21}^{0}(m)=\sum_{k=-\infty}^{\infty} l(k) a_{11}^{(0)}(m-k) \\
& a_{21}^{1}(m)=\sum_{k=-\infty}^{\infty} l(k) a_{11}^{(1)}(m-k), \\
& a_{21}^{2}(m)=\sum_{k=-\infty}^{\infty} l(k) a_{11}^{(2)}(m-k)
\end{aligned}
$$

where $\alpha_{21}^{v}, \alpha_{11}^{v}, .=0,1,2$ are the connection coefficients of the base wavelets and can be evaluated analytically [7]. In general, these equations may be over-determined. We therefore solve for $l(k)$, the sequence that satisfies (20) in the least square sense. Using this, we compute the lifted discrete filters as follows [10]:

$$
\begin{aligned}
& \alpha^{l}(n)=\alpha(n)+\sum_{k=-\infty}^{\infty} \beta(n-2 k) \hat{l}(-k), \\
& \tilde{\beta}^{l}(n)=\tilde{\beta}(n)-\sum_{k=-\infty}^{\infty} \tilde{\alpha}(n-2 k) \hat{l}(k),
\end{aligned}
$$

and $(\alpha, \beta, \widetilde{\alpha}, \widetilde{\beta})$ is replaced with $\left(\alpha^{l}, \beta, \widetilde{\alpha}, \widetilde{\beta}^{l}\right)$.

In this study, we chose to design our wavelet based on the biorthogonal spline wavelet bior3.3 [11]. Figure 1 shows the scaling and wavelet functions of the bior3.3 wavelet, and following the procedure above its lifted version, the bior3.3_L wavelet. In order to evaluate how well the bior3.3_L wavelet approximate threefold ortogonality we looked at the ratio between the off diagonal blocks in $\mathbf{Q}$ and the diagonal blocks in $\mathbf{Q}$ :

$$
\left.T F O=\underset{s \neq t, j \neq l}{\operatorname{mean}} \underset{\text { Frobenius }}{\operatorname{norm}}\left(Q_{j l}^{s t}\right)\right) / \underset{s=t, j=l}{\operatorname{mean}}\left(\underset{\text { Frobenius }}{\operatorname{norm}}\left(Q_{j l}^{s t}\right)\right)
$$

Computing the TFO for the bior3.3_L wavelet resulted in a value of 0.02 (Note that in the case of threefold orthogonal wavelet $T F O=0$.)

\section{EXPERIMENTAL RESULTS}

The performance of the registration algorithm was evaluated in a two-dimensional setting. Ten mouse brain images were studied: one, the reference brain image, was used to construct a three dimensional brain volume atlas (see [12]) and the other nine brain images (generated using a technique described in [13]) were the test brain images. From each test brain, a coronal section located in the olfactory bulbs was extracted together with its corresponding section in the atlas brain. This matching of a reference section to its test section was done manually using the MacOStat software package [14, 15]. Figure 2 shows one pair of reference and corresponding test sections. The high morphological variability between corresponding sections is clearly evident. Next, nineteen internal anatomical structures (see Table 1 for structures' nomenclatures based on the Franklin and Paxinos atlas [16]) were manually delineated on all sections. 


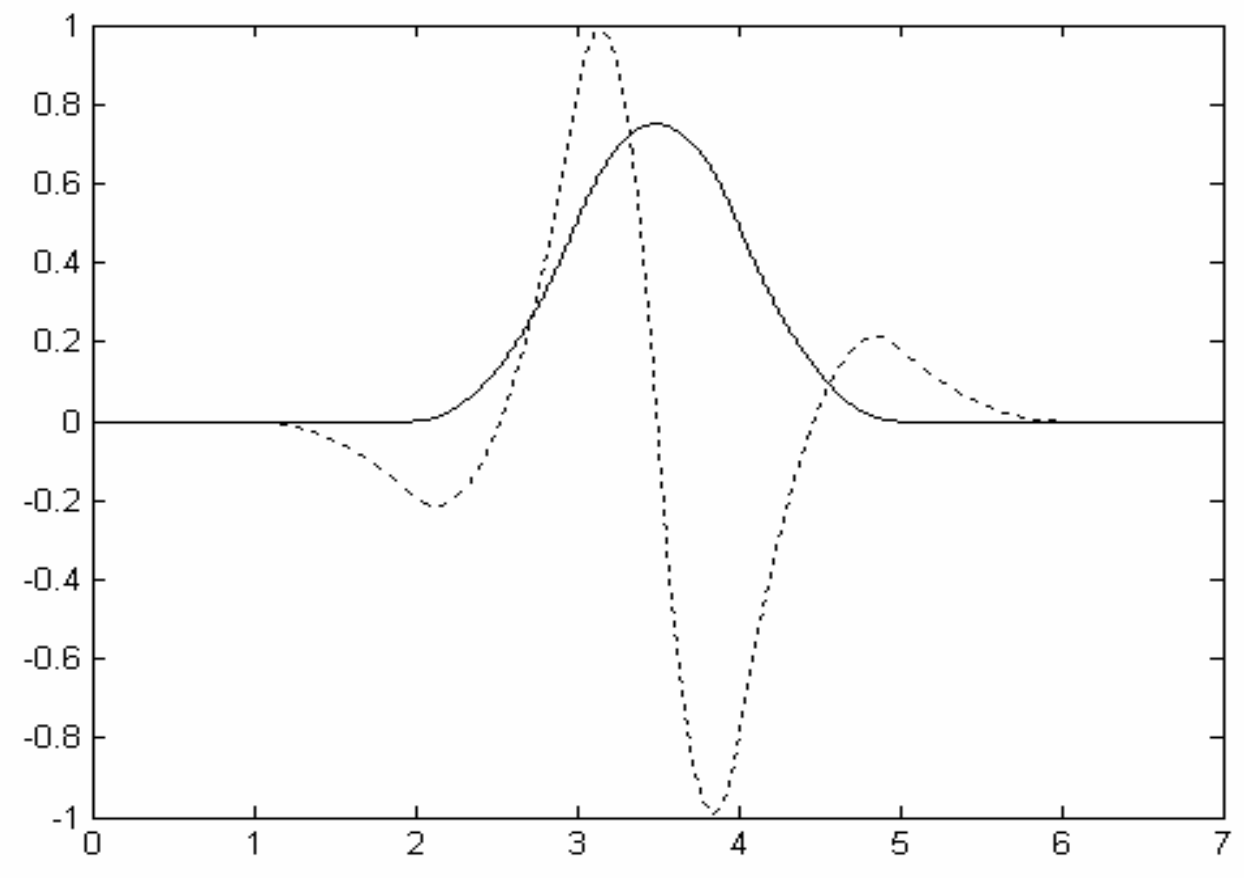

(a)

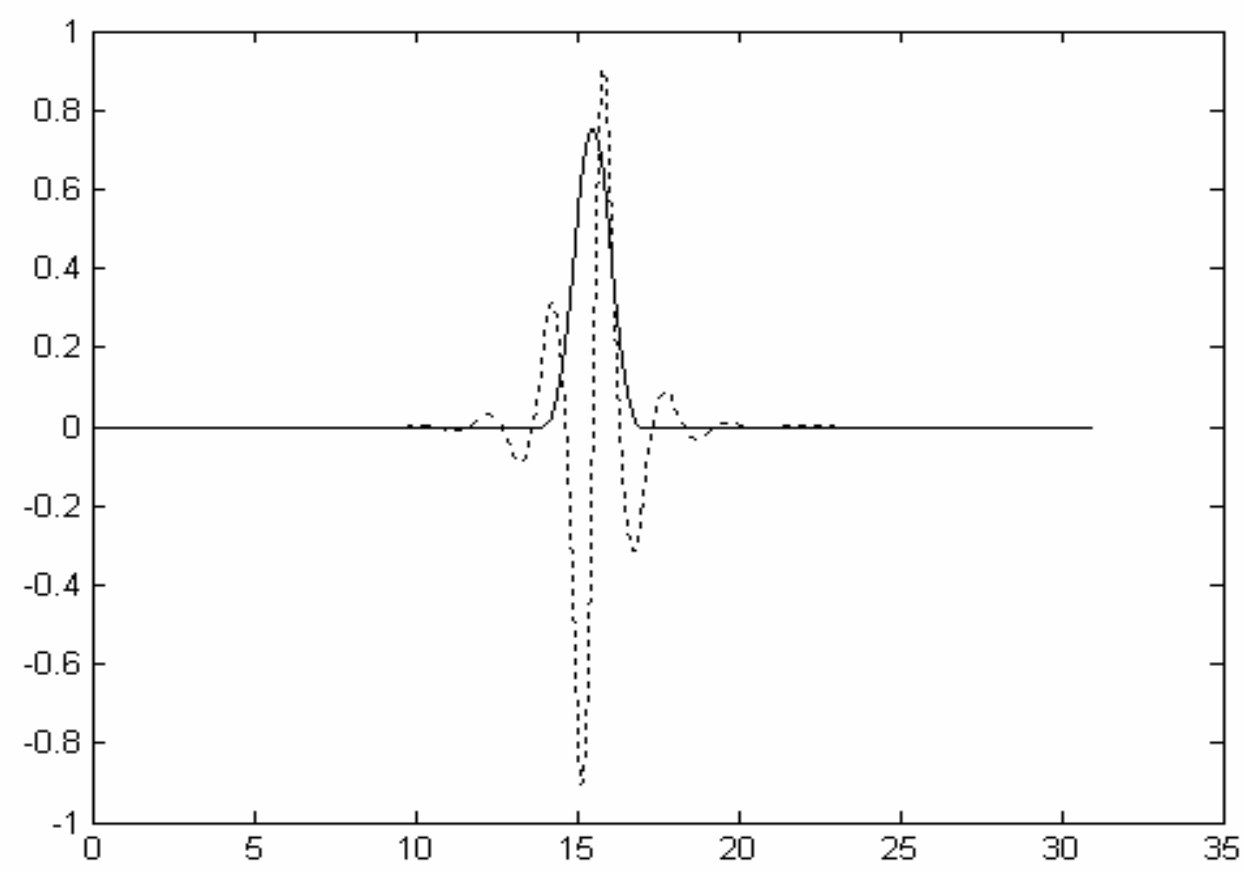

(b)

Figure 1. The scaling and wavelet functions of (a) the bior3.3 wavelet, and (b) the lifted bior3.3_L wavelet. 

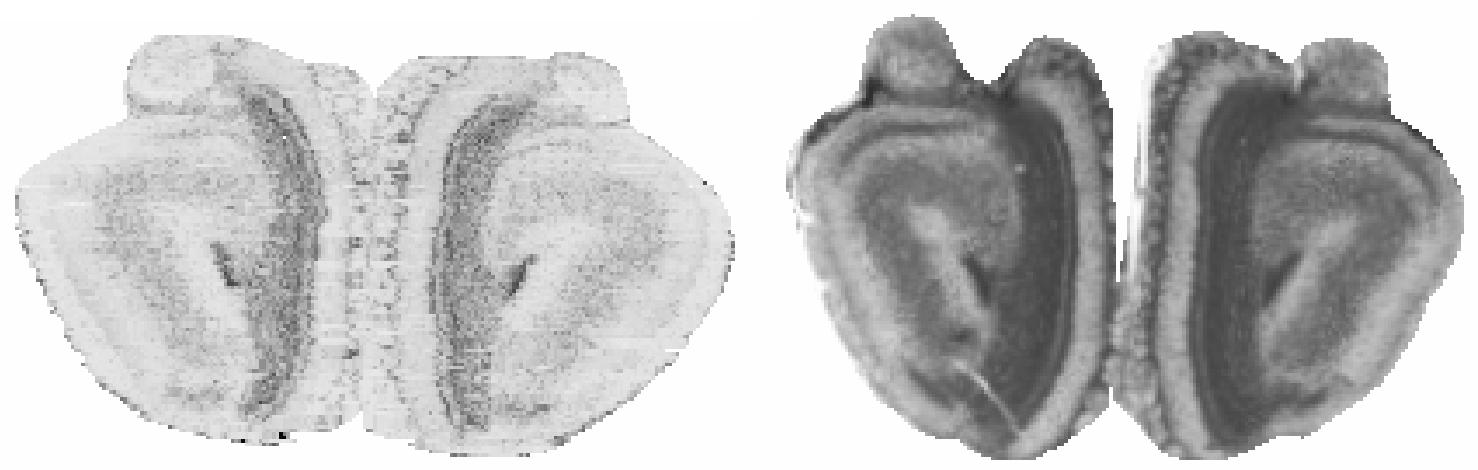

Figure 2: Olfactory bulbs matching coronal sections from the atlas (left) and an experimental brain (right).

Using the wavelet-based alignment algorithm, each section was aligned to its matching reference section. To do so a subset of the manually delineated contours were used to guide the alignment process and the remaining ones were used for performance evaluation, as shown in Figure 3. We first aligned corresponding sections linearly and then recovered the nonlinear deformation everywhere within a support of 256 by 256 using the bior33_L wavelet and including resolution levels 7 through 3 .
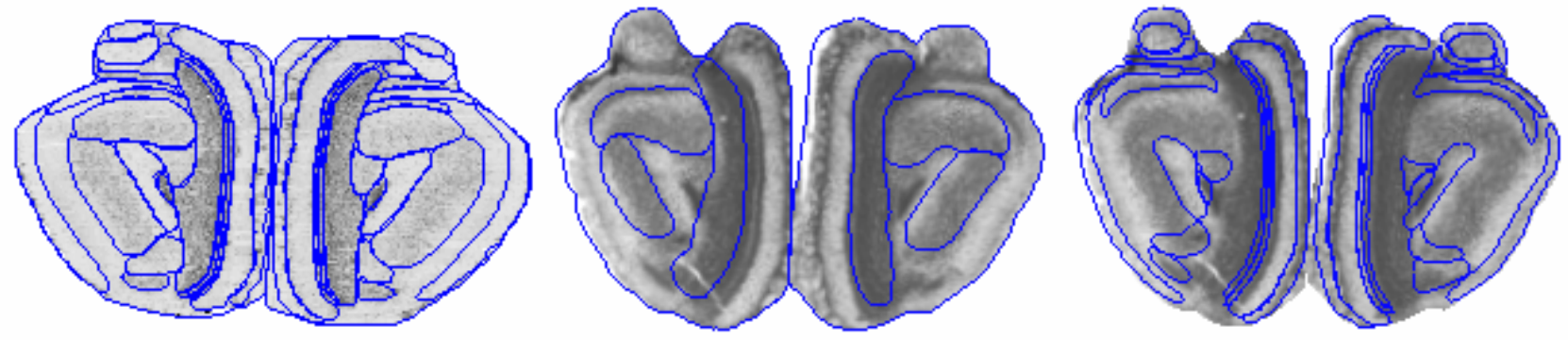

Figure 3: A reference section from the olfactory bulbs with all the structures' contours manually delineated (left), and its corresponding test section (center and right). The contours delineated on the section at the center were used to guide registration (GR) while the contours delineated on the section at the right were used to validate registration (VR).

Two performance measures were computed for each structure and averaged across the nine brains: error (average residual contour distance between corresponding contours), and overlap (the area of the overlap of corresponding structures divided by the area of the reference structure). In addition, the mutual information (MI) along the structures' contours was computed as described in [17]. The error, overlap, and MI values were computed for the data before the nonlinear alignment (after an affine transform was applied) and after the wavelet alignment.

Table 1 contains the results (the structures that guide the registration are denoted in bold). The three rows at the bottom of tables 1 show averages over all structures weighted by structure area. We tabulate averages for registration guiding (RG) structures, for registration validating (RV) structures, and for all structures (Total). As can be seen from Table 1, the reduction of the error was substantial. On average for all the sections combined the errors were reduced from 3.6 to 0.8 pixels, the average increase in the overlap between corresponding structures was from $75 \%$ to $89 \%$, and the average increase in MI was $17 \%$. 
Table 2 demonstrates the scalability when aligning a test section from one brain to the corresponding section from the atlas. The error residual, the increase in corresponding structures overlap, and the increase in image mutual information are reported in addition to the registration parameters' number and the running time. The tradeoff between accuracy and complexity is apparent. The running times reported in Table 2 are based on algorithm implementation using Matlab (version 6.1) on a Pentium IV computer (1.9 GHz, 2G Byte RAM).

Table 1 The average error and overlap ratio of before and after wavelet-based alignment

\begin{tabular}{|l|l|c|c|c|c|}
\hline \multicolumn{2}{|l|}{} & \multicolumn{2}{c|}{$\begin{array}{c}\text { Before } \\
\text { Registration }\end{array}$} & \multicolumn{2}{c|}{$\begin{array}{c}\text { After } \\
\text { Registration }\end{array}$} \\
\hline The Olfactory Bulbs Anatomical Structures & Error & Overlap & Error & Overlap \\
\hline GrO & granule layer, olfactory bulb & $\mathbf{2 . 7 4}$ & $\mathbf{0 . 8 0}$ & $\mathbf{0 . 2 5}$ & $\mathbf{0 . 9 8}$ \\
\hline E/OV & ependyma \& olfactory ventricle & 2.12 & 0.40 & 1.63 & 0.54 \\
\hline aci & anterior commissure, intrabulbar & 2.24 & 0.47 & 1.08 & 0.66 \\
\hline IPl & internal plexiform layer, olfactory bulb & 1.87 & 0.26 & 0.59 & 0.64 \\
\hline Mi & mitral cell layer, olfactory bulb & 2.05 & 0.28 & 0.91 & 0.59 \\
\hline EPl & external plexiform layer, olfactory bulb & 2.69 & 0.70 & 1.79 & 0.83 \\
\hline Gl & glomerular layer, olfactory bulb & 2.38 & 0.49 & 2.14 & 0.63 \\
\hline AOE & anterior olfactory nucleus, external & 4.46 & 0.40 & 2.66 & 0.72 \\
\hline vn & vomeronasal nerve & 3.83 & 0.42 & 3.75 & 0.48 \\
\hline EPlA & external plexiform layer accessory olfactory bulb & 3.60 & 0.30 & 2.63 & 0.40 \\
\hline MiA & mitral cell layer, olfactory bulb & 4.84 & 0.33 & 3.47 & 0.48 \\
\hline AOL & anterior olfactory nucleus, lateral & $\mathbf{3 . 4 6}$ & $\mathbf{0 . 7 5}$ & $\mathbf{0 . 2 1}$ & $\mathbf{0 . 9 7}$ \\
\hline AOM & anterior olfactory nucleus, medial & 3.74 & 0.44 & 2.69 & 0.59 \\
\hline AOD & anterior olfactory nucleus, dorsal & $\mathbf{3 . 6 7}$ & $\mathbf{0 . 7 3}$ & $\mathbf{0 . 2 9}$ & $\mathbf{0 . 9 5}$ \\
\hline dlo & dorsolateral olfactory tract & 4.35 & 0.18 & 2.73 & 0.40 \\
\hline GlA & glomerular layer, accessory olfactory bulb & 3.81 & 0.50 & 3.05 & 0.63 \\
\hline lo & lateral olfactory tract & 2.47 & 0.40 & 2.24 & 0.62 \\
\hline AOV & anterior olfactory nucleus, ventral & 3.71 & 0.46 & 2.08 & 0.75 \\
\hline OB & olfactory bulb (external structure) & $\mathbf{4 . 0 6}$ & $\mathbf{0 . 9 0}$ & $\mathbf{0 . 2 4}$ & $\mathbf{0 . 9 9}$ \\
\hline & & 4.09 & 0.90 & 0.24 & 0.99 \\
\cline { 2 - 6 } & & 2.91 & 0.58 & 2.00 & 0.67 \\
\hline & & 3.55 & 0.75 & 0.79 & 0.89 \\
\hline
\end{tabular}

Table 2: Average error, overlap and mutual information increase, and algorithm complexity for the registration of corresponding sections from one brain sample and including different resolution levels

\begin{tabular}{|c|c|c|c|c|c|}
\hline Res. Levels & Error (pixels) & Overlap (\%) & MI increase (\%) & Parameters No. & Time (secs) \\
\hline 7 & 2.26 & 0.82 & 8 & $2 \times 4 \times 4$ & 96 \\
\hline 7 through 6 & 1.83 & 0.86 & 10 & $+2 \times 3 \times \mathbf{1 6}$ & 189 \\
\hline 7 through 5 & 1.10 & 0.90 & 15 & $+2 \times 3 \times 64$ & 279 \\
\hline 7 through 4 & 0.78 & 0.92 & 16 & $+2 \times 3 \times 179$ & 384 \\
\hline 7 through 3 & 0.66 & 0.92 & 17 & $+2 \times 3 \times 497$ & 570 \\
\hline
\end{tabular}

\section{CONCLUSION}

Registration of images of histological material is often complicated by the large size of the datasets involved. The problem is simplified in the described algorithm. The wavelet representation allowed for a multi-resolution decomposition of the deformation field. Specifically, the wavelet threefold orthogonality assumption enabled problem formulation simplification. 
The performance of the wavelet-based registration algorithm was tested on murine brain sections. A subset of external and internal structures were used to guide the registration. The distance of test contours from their corresponding reference contours as well as the deformation elastic energy were minimized. The alignment validation yielded the following results: (1) on average the residual distance between corresponding structures' contours resulted in a 4.5 fold alignment improvement over linear registration, (2) the average increase in overlap between corresponding structures was $14 \%$, and (3) the average increase in mutual information was $17 \%$.

\section{REFERENCES}

1. R. Bajcsy and S. Kovacic, "Multi-resolution Elastic Matching," Computer Vision, Graphics and Image Processing, vol. 46, pp. 1-21, 1989.

2. $\quad$ C. Broit, "Optimal registration of deformed images," : Univ. Pennsylvania, 1981.

3. Y. Amit, "A Nonlinear Variational Problem for Image Matching," SIAM J. Scientific Comput., vol. 15, pp. 207224, 1994.

4. T. R. Downie, L. Shepstone, and B. W. Silverman, "A Wavelet Based Approach to Deformable Templates," presented at Image Fusion and Shape Variability Techniques, Leeds, UK, 1996.

5. Y. T. Wu, L. F. Chen, P. L. Lee, T. C. Yeh, and J. C. Hsieh, "Discrete Signal Matching Using Coarse-to-fine Wavelet Basis Functions," Pattern Recoginition, vol. 36, pp. 171-192, 2003.

6. J. Deubler and J. C. Olivo, "A Wavelet Based Multiresolution Method to Automatically Register Images," Journal of Mathematical Imaging and Vision, vol. 7, pp. 199-209, 1997.

7. S. Gefen, "Wavelet-based Nonlinear Multi-dimensional Registration," in Doctoral thesis in Electrical and Computer Engineering. Philadelphia: Drexel University, 2002.

8. D. W. Marquardt, "An Algorithm for Least-squares Estimation of Nonlinear Parameters," J. Soc. Indust. Appl. Math., vol. 11, pp. 431-441, 1963.

9. D. Kozinska, O. J. Tretiak, J. Nissanov, and C. Ozturk, "Multidimentional Alignment Using the Euclidean Distance Transform," Graphical Models and Image Processing, vol. 59, pp. 373-387, 1997.

10. W. Sweldens, "The Lifting Scheme: A Custom - Design Construction of Biorthogonal Wavelets," J. of App. and Comput. Harmonic Analysis, vol. 3, pp. 186-200, 1996.

11. I. Daubechies, Ten Lectures on Wavelets. Philadelphia: Society for Industrial and Applied Mathematics (SIAM), 1997.

12. J. Nissanov, J. Eilbert, O. J. Tretiak, S. Gefen, S. Schremmer, C. Gustafson, and L. Bertrand, "3D Atlases Bridges Between Neurogenomics and Neuroanatomy," presented at IEEE International Symposium on Biomedical Imaging, Washington DC, 2002.

13. G. D. Rosen, A. G. Williams, J. A. Capra, M. T. Connolly, B. Cruz, L. Lu, D. C. Airey, K. Kulkarni, and R. W. Williams, "The Mouse Brain Library @ www.mbl.org," presented at Int Mouse Genome Conference, 2000.

14. C. Gustafson, O. Tretiak, L. Bertrand, and J. Nissanov, "Design and Implementation of Software for Assembly and Browsing of 3D Brain Atlases," Computer Methods and Programs in Biomedicine, In press.

15. G. D. Rosen, N. T. L. Porte, B. Diechtiareff, C. J. Pung, J. Nissanov, C. Gustafson, L. Bertrand, S. Gefen, Y. Fan, O. J. Tretiak, K. F. Manly, M. R. Park, A. G. Williams, T. Connolly, J. A. Capra, and R. W. Williams, "Informatics Center for Mouse Genomics: The Dissection of Complex Traits of the Nervous System," Bioinformatics, In press.

16. K. B. J. Franklin and G. Paxinos, The Mouse Brain in Streotaxic Coordinates: Academic Press, 1997.

17. F. Maes, A. Collignon, D. Vandermeulen, G. Marchal, and P. Suetens, "Multimodality Image Registration by Maximization of Mutual Information," IEEE Transactions on Medical Imaging, vol. 16, pp. 187-198, 1997. 